CTP TAMU-19/96

hep-th/9605077

\title{
Intersecting M-branes and Bound States
}

\author{
N. Khviengia, Z. Khviengia, H. Lü ${ }^{\dagger}$, and C.N. Pope ${ }^{\dagger}$ \\ Center for Theoretical Physics \\ Texas A\&M University, College Station, Texas 77843
}

\begin{abstract}
$\underline{\text { ABSTRACT }}$
In this paper, we construct multi-scalar, multi-center $p$-brane solutions in toroidally compactified M-theory. We use these solutions to show that all supersymmetric $p$-branes can be viewed as bound states of certain basic building blocks, namely $p$-branes that preserve $1 / 2$ of the supersymmetry. We also explore the M-theory interpretation of $p$-branes in lower dimensions. We show that all the supersymmetric $p$-branes can be viewed as intersections of M-branes or boosted M-branes in $D=11$.
\end{abstract}

\footnotetext{
${ }^{\dagger}$ Research supported in part by DOE Grant DE-FG05-91-ER40633
} 


\section{Introduction}

The construction and classification of $p$-brane solitons has been increasingly recognised to be important for gaining insights into the non-perturbative structures underlying string theory and M-theory. In particular, with the developing interest in eleven-dimensional M-theory, it is of interest to try to classify and interpret all its the extended-object solutions. In $D=11$ itself, there are only two isotropic solutions, namely the elementary membrane [1] and the solitonic 5-brane [2]. As one descends through the dimensions to obtain lower-dimensional supergravities by dimensional reduction, a plethora of isotropic $p$-brane solutions arises. A fairly complete classification of these in toroidally-compactified M-theory was given in [3].

There are several possible approaches that can be taken in order to try to organise these lower-dimensional $p$-branes into a more coherent picture. One interesting idea, which was applied to black-hole solutions in $D=4$, was to describe the various inequivalent supersymmetric black holes as bound states of a fundamental "building block," namely the single-charge $a=\sqrt{3}$ black hole that preserves $1 / 2$ of the supersymmetry 四, 同, 6 , 7. 8. Exploiting the fact that extremal black holes satisfy a "no-force" condition, which allows the construction of multi-center black hole solutions, it was shown in [8] that all the other supersymmetric black holes in $D=4$, corresponding to configurations where multiple charges are non-zero, could be obtained as bound states in the multi-center $a=\sqrt{3}$ solutions.

One of the purposes of the present paper is to extend the bound-state discussion to a general setting. Specifically, we shall show that all supersymmetric $p$-brane solutions in M-theory can be described in terms of certain fundamental $p$-branes that all preserve $1 / 2$ of the supersymmetry. To do this, we first construct general classes of multi-center supersymmetric $p$-branes. In order to encompass as broad a class of solutions as possible, we consider multi-scalar solutions, in which generically there are $N$ non-vanishing dilatonic scalar fields and $N$ independent charges carried by different field strengths, and show how to construct multi-center generalisations in which each of the the multi-centers for each of the $N$ charges can be located independently at different positions in the transverse space. In particular, when the locations of all the charges are coincident the solution reduces to an isotropic multi-scalar soliton [9]. Furthermore, if in addition all the charges are set equal, they become single-scalar solutions. There are also a few examples of extremal $p$-branes that are non-supersymmetric, but which are related to supersymmetric solutions simply by reversing the signs of certain charges [9, 10]. These can also be viewed as bound states of the same basic building blocks. It should also be emphasised, however, that there are many more isotropic single-scalar $p$-brane solutions [3] that, although extremal, are neither 
supersymmetric nor related by sign reversals to supersymmetric solutions. Although they can be generalised to multi-center solutions [16], they do not admit suitable multi-scalar generalisations that would enable them to be viewed as bound states.

Another approach to classifying the lower-dimensional $p$-brane solutions in M-theory is to retrace the steps in the Kaluza-Klein dimensional reduction of the supergravity theory, and thereby look at the oxidations of the $p$-branes back to $D=11$. The forms of the resulting configurations depend crucially on the details of which particular field strengths in the lower dimension are involved in the construction of the $p$-brane solution. In certain simple cases, the oxidised solution simply becomes the isotropic membrane or 5-brane of the eleven-dimensional theory. More commonly, however, the oxidised solution exhibits less than maximal isotropy either in the world-volume dimensions of the resulting extended object, or in the transverse space, or both. Some of these configurations can be interpreted as describing intersections of membranes or 5-branes in $D=11$ [11, 12, 13, 14, 15, 16]. If the field strengths participating in the lower-dimensional solution include those that come from the $D=11$ vielbein under Kaluza-Klein dimensional reduction, this anisotropy will include off-diagonal terms in the $D=11$ metric describing some kind of twist, or non-trivial fibration, in the spacetime. In some cases, these off-diagonal terms have been interpreted as describing momentum flow [17] along the spatial dimensions of the extended object. In this paper, we shall also explore some of these oxidation pathways in more detail, and show how some of the lower-dimensional $p$-branes may be interpreted back in $D=11$.

\section{Multi-scalar multi-center $p$-branes}

Let us begin by constructing the multi-scalar, multi-center solutions. The relevant bosonic part of the supergravity Lagrangian, describing $N$ dilatonic scalar fields and $N n$-index antisymmetric tensor field strengths is given by [9]

$$
\mathcal{L}=e R-\frac{1}{2} e(\partial \vec{\phi})^{2}-\frac{1}{2 n !} e \sum_{\alpha=1}^{N} e^{-\vec{a}_{\alpha} \cdot \vec{\phi}}\left(F^{\alpha}\right)^{2}
$$

The metric ansatz is given by

$$
d s^{2}=e^{2 A} d x^{\mu} d x^{\nu} \eta_{\mu \nu}+e^{2 B} d y^{m} d y^{m}
$$

where $x^{\mu}(\mu=0, \ldots, d-1)$ are the coordinates of the $(d-1)$-brane world volume, and $y^{m}$ are the coordinates of the $(D-d)$-dimensional transverse space. The functions $A$ and $B$, as well as the dilatonic scalars, depend on the transverse coordinates $y^{m}$ only. For each 
$n$-index field strength $F^{\alpha}$, one can construct an elementary $(n-2)$-brane carrying electric charges with world volume dimension $d=n-1$, or a solitonic $(D-n-2)$-brane carrying magnetic charges with $d=D-n-1$. In this paper, we shall consider cases for $N \geq 2$ where the field strengths all carry either electric or magnetic charges. For the case $N=1$, we shall also consider the dyonic string in $D=6$.

We shall first consider the case where the field strengths carry electric charges, for which

$$
F_{m \mu_{1} \cdots \mu_{n-1}}^{\alpha}=\epsilon_{\mu_{1} \cdots \mu_{n-1}} \partial_{m} e^{C_{\alpha}}
$$

where the functions $C_{\alpha}$ depend only on the transverse-space coordinates $y^{m}$. It is straightforward to obtain the equations of motion following from the Lagrangian (11), and to substitute the ansätze for the field strength and the metric. As in the case of the isotropic multi-scalar solutions, one may obtain simple solutions by making a further ansatz, namely $d A+\tilde{d} B=0$. Here, however, we do not assume isotropicity in the transverse space. In order to obtain multi-center solutions, we first show that the resulting equations of motion can be cast into a linear form. The remaining equations of motion are given by

$$
\begin{aligned}
& \partial_{m} \partial_{m} \varphi_{\alpha}=\frac{1}{2} \sum_{\beta} M_{\alpha \beta} S_{m}^{\beta} S_{m}^{\beta}, \\
& \partial_{m} \partial_{m} A=\frac{\tilde{d}}{2(D-2)} \sum_{\alpha} S_{m}^{\alpha} S_{m}^{\alpha}, \\
& d(D-2) \partial_{m} A \partial_{n} A+\frac{1}{2} \tilde{d} \sum_{\alpha, \beta}\left(\partial_{m} \varphi_{\alpha}\right)\left(\partial_{n} \varphi_{\beta}\right)=\frac{1}{2} \tilde{d} \sum_{\alpha} S_{m}^{\alpha} S_{n}^{\alpha}, \\
& \partial_{m} \partial_{m} C_{\alpha}+\partial_{m} C_{\alpha}\left(\partial_{m} C_{\alpha}-2 d \partial_{m} A-\partial_{m} \varphi_{\alpha}\right)=0,
\end{aligned}
$$

where we have made following definitions

$$
\begin{aligned}
\varphi_{\alpha} & =\vec{a}_{\alpha} \cdot \vec{\phi}, \quad M_{\alpha \beta}=\vec{a}_{\alpha} \cdot \vec{a}_{\beta}, \\
S_{m}^{\alpha} & =e^{-\frac{1}{2} \varphi-d A} \partial_{m} e^{C_{\alpha}} .
\end{aligned}
$$

It follows from equations (田) and (5) that it is natural to solve for $A$ by taking

$$
A=\frac{\tilde{d}}{D-2} \sum_{\alpha, \beta}\left(M^{-1}\right)_{\alpha \beta} \varphi_{\alpha} .
$$

In the case of isotropic $p$-branes, the supersymmetric multi-scalar $p$-brane solutions arise if the dot products $M_{\alpha \beta}$ of the dilaton vectors $\vec{a}_{\alpha}$ satisfy [9]

$$
M_{\alpha \beta}=4 \delta_{\alpha \beta}-\frac{2 d \tilde{d}}{D-2},
$$


where $\tilde{d}=D-d-2$. It was shown in [18] that the equations of motion describing supersymmetric multi-scalar $p$-brane solutions can be reduced to a set of $N$ Liouville equations with vanishing Hamiltonian. Since these solutions are supersymmetric and hence extremal, the no-force condition is satisfied, and we can generalise them to multi-center solutions. On the other hand, for the dot products that do not satisfy (10), the equations of motion can be reduced to Toda-like equations, whose solutions are intrinsically non-extremal [18]. We do not expect that such solutions admit multi-scalar generalisations.

The inverse of the matrix $M_{\alpha \beta}$ (10) is given by

$$
M_{\alpha \beta}^{-1}=\frac{1}{4} \delta_{\alpha \beta}+\frac{d \tilde{d}}{4(2 D-4-d \tilde{d} N)} .
$$

For $N \geq 2$, it is singular in two cases, namely for $N=3$ black holes or strings in $D=5$, and for $N=4$ black holes in $D=4$. We shall proceed for now by assuming $M_{\alpha \beta}$ is invertible, and return to these singular cases later, when we shall see that the general solution also solves the equations in these cases. Since $M_{\alpha \beta}$ is given by (10), it follows from (4) and (5) that the equations diagonalise with respect to the index $\alpha$, i.e. they take the form $\partial_{m} \partial_{m}\left(\frac{1}{2} \varphi_{\alpha}+d A\right)=S_{m}^{\alpha} S_{m}^{\alpha}$ for each value of $\alpha$. These equations admit solutions for which $S_{m}^{\alpha}=\partial_{m}\left(\frac{1}{2} \varphi_{\alpha}+d A\right)$, implying that

$$
\partial_{m} \partial_{m} e^{-\frac{1}{2} \varphi_{\alpha}-d A}=0
$$

and $C_{\alpha}=\frac{1}{2} \varphi_{\alpha}+d A$. It can easily be verified that the remaining two equations (6) and (7) are now also satisfied. Thus we have reduced the original equations of motion to a set of $N$ linear equations (12), which admit multi-centered solutions

$$
e^{-\frac{1}{2} \varphi_{\alpha}-d A} \equiv H_{\alpha}=1+\sum_{i} \frac{k_{i}^{\alpha}}{\left|\vec{y}-\vec{y}_{i}^{\alpha}\right| \tilde{d}},
$$

where $k_{i}^{\alpha}$ is the $i$ 'th charge for the $\alpha^{\prime}$ th field strength, located at $\vec{y}_{i}^{\alpha}$ in the transverse space. Note that the charges are independent in number, size, and location, for each harmonic function $H_{\alpha}$. The metrics of the elementary multi-center multi-scalar $p$-brane solutions are therefore given by

$$
\begin{aligned}
d s^{2} & =e^{2 A} d x^{\mu} d x^{\nu} \eta_{\mu \nu}+e^{2 B} d y^{m} d y^{m} \\
e^{2 A} & =\prod_{\alpha=1}^{N}\left(1+\sum_{i} \frac{k_{i}^{\alpha}}{\left|\vec{y}-\vec{y}_{i}^{\alpha}\right|^{\tilde{d}}}\right)^{-\frac{\tilde{d}}{D-2}} \\
e^{2 B} & =\prod_{\alpha=1}^{N}\left(1+\sum_{i} \frac{k_{i}^{\alpha}}{\left|\vec{y}-\vec{y}_{i}^{\alpha}\right|^{\tilde{d}}}\right)^{\frac{d}{D-2}} .
\end{aligned}
$$


The above discussion assumed that the matrix $M_{\alpha \beta}$ was invertible. The solutions contain $N$ dilatonic scalar fields, which is consistent with having independent charges for the $N$ field strengths. If the matrix $M_{\alpha \beta}$ is singular, which can arise in the two cases $N=3, D=5$ and $N=4, D=4$ that we discussed above, the generic form of the metric (14) continues to solve the equations of motion. However, in this case it follows from (9) and (13) that the dilatonic scalar fields are no longer independent, but satisfy $\sum_{\alpha} \varphi_{\alpha}=0$. Thus the solutions in these two cases contain $N$ independent charges, but only $(N-1)$ dilatonic scalar fields.

Having obtained the explicit elementary solutions, it is now straightforward to construct the solitonic solutions. The solitonic ansatz for the field strength is given by

$$
F_{m_{1} \cdots m_{n}}^{\alpha}=-\epsilon_{m_{1} \cdots m_{n} p} \partial_{p} \sum_{i} \frac{k_{i}^{\alpha}}{\left|\vec{y}-\vec{y}_{i}^{\alpha}\right|^{\tilde{d}}} .
$$

The solitonic solutions can be obtained from the corresponding elementary ones by making the replacement $\varphi_{\alpha} \rightarrow-\varphi_{\alpha}$. It is worth remarking that the terms "elementary" and "solitonic" are being used with respect to the field strengths given in the Lagrangian (1). In fact in $D=2 n$ dimensions, some of the field strengths in the Lagrangian (1) can be the duals of the original field strengths of the same rank in the supergravity theory. Thus in terms of the original fields, the solutions may carry both electric and magnetic charges. Such solutions were called dyonic $p$-branes of the first type in [3]. The solutions (14) also include multi-center dyonic strings of the second type in $D=6$, where a single 3 -form field strength carries both electric and magnetic charges. Note that the electric and magnetic charges $k_{i}^{\alpha}$ in the multi-center harmonic functions can be located at different places.

\section{$3 \quad$ Extremal $p$-branes as bound states}

In the previous section, we obtained the general supersymmetric multi-scalar multi-center $p$-brane solutions. When all the charges are equal and located in the same point in the transverse space, the solutions reduce to isotropic single-scalar solutions for the Lagrangian

$$
e^{-1} \mathcal{L}=R-\frac{1}{2}(\partial \phi)^{2}-\frac{1}{2 n !} e^{-a \phi} F^{2}
$$

where the constant $a$ can be parameterised as $a^{2}=\Delta-2 d \tilde{d} /(D-2)$ with $\Delta=4 / N$. The supersymmetric isotropic single-scalar $p$-branes in toroidally-compactified M-theory in all dimensions were classified in [3]. It follows from our results in section 2 that the multiplycharged solutions can be regarded as bound states of singly-charged $p$-branes. Consider a $p$-brane in which each field strength $F^{\alpha}$ carries a single charge $k^{\alpha}$ located at $\vec{y}^{\alpha}$, for which 
the metric is given by

$$
\begin{aligned}
d s^{2} & =e^{2 A} d x^{\mu} d x^{\nu} \eta_{\mu \nu}+e^{2 B} d y^{m} d y^{m} \\
e^{2 A} & =\prod_{\alpha=1}^{N}\left(1+\frac{k_{\alpha}}{\left|\vec{y}-\vec{y}_{\alpha}\right|^{\tilde{d}}}\right)^{-\frac{\tilde{d}}{D-2}} \\
e^{2 B} & =\prod_{\alpha=1}^{N}\left(1+\frac{k_{\alpha}}{\left|\vec{y}-\vec{y}_{\alpha}\right|^{\tilde{d}}}\right)^{\frac{d}{D-2}} .
\end{aligned}
$$

The metric approaches that of a singly-charged $p$-brane in the vicinity of each of the locations $\vec{y}^{\alpha}$. If these locations are chosen to be coincident, the solution reduces to a multi-scalar $p$ brane solution with independent charge parameters for the $N$ field strengths. If in addition the charges are chosen to be equal, this solution then reduces to a single-scalar solution with $\Delta=4 / N$. Thus these multiply-charged single-scalar or multi-scalar $p$-brane solutions can be viewed as bound states of singly-charged $p$-branes. In other words, all single-scalar supersymmetric $p$-branes with $\Delta=4 / N=2,4 / 3,1, \ldots$, as well as all their multi-scalar generalisations, can be described as bound states of basic $\Delta=4$ single-charge building blocks (each of which by itself would preserve $1 / 2$ of the supersymmetry). In addition, there are certain non-supersymmetric $p$-branes with $\Delta=4 / N$ for $N \geq 4$, which are related to supersymmetric ones simply by reversing the signs of certain charges [9, 10]. These too can be described as bound states of the same $\Delta=4$ building blocks. (Note however that the non-supersymmetric extremal $p$-branes with $\Delta \neq 4 / N$ do not appear to admit a bound-state interpretation, since the necessary multi-scalar generalisations do not exist.) Of course, since all of the above $p$-brane examples are constructed from configurations that obey a no-force condition, they are bound states with zero binding energy.

It is worth remarking that the solutions (17) also include the multi-center dyonic string in $D=6$, where the electric charge and magnetic charges are located at different points in the transverse space. If the two location coincide, it becomes the standard isotropic dyonic string. Thus the isotropic dyonic string [19], including the self-dual [20] and quasi-anti-selfdual [3, 21] limits, is a bound state of the $\Delta=4$ electric string and the $\Delta=4$ magnetic string.

All the $\Delta=4$ building blocks are singular-dilaton solutions, in the sense that the dilaton field diverges at the horizon. These divergences indicate that the classical tree-

\footnotetext{
${ }^{1}$ There do in fact exist some $\Delta=4$ solutions that do not involve any dilaton, and hence avoid this kind of singular behaviour, namely the M-branes (membrane [1] and 5-brane [2]) in $D=11$, and the self-dual 3-brane in $D=10$ type IIB supergravity 22, 23. However, these solutions cannot be used to construct bound states, because, obviously, they do not admit the necessary multi-scalar generalisations, and hence we shall not regard them as building blocks for bound states.
} 
level approximation is insufficient, and that string and world-sheet loop corrections can be expected to modify the solutions significantly. It is interesting, however, that some of the bound states built from these singular-dilaton building blocks are nevertheless regulardilaton solutions. Four such cases are known, namely the dyonic string (with two charges) in $D=6$, the 3 -charge black hole in $D=5$ (or its string dual), the 4-charge black hole in $D=4$, and a special dyonic black hole (also with four charges) [3] in $D=4$. In these solutions, there are a total of $(N-1)$ non-vanishing dilatonic scalar fields, where $N$ is number of the charges that the solution carries. These scalar fields are regular at the horizon, and throughout the whole spacetime. If the charges are set equal, the dilatonic scalar field decouples, and the solutions reduce to the self-dual string with $\Delta=2$ in $D=6$, the Reissner-Nordstrøm black holes with $\Delta=4 / 3$ or $\Delta=1$ in $D=5$ or $D=4$ respectively, and the special dyonic black hole with $\Delta=1$ in $D=4$ [3]. These solutions are non-dilatonic, and are analogous to M-branes in $D=11$ or the self-dual 3-brane in $D=10$.

Since these four bound states have dilatons that are regular throughout the spacetime, they may not be appreciably modified by quantum corrections. Thus the string theory may provide a microscopic interpretation of the entropy for these solutions. In particular, the Reissner-Nordstrøm black holes in $D=5$ and $D=4$ can be oxidised into a boosted dyonic string in $D=6$ [17] and a boosted 3-charge string in $D=5$ respectively. Both these strings can be viewed as Dirichlet strings carrying momentum, and hence their microscopic states can be counted. Since the entropy per unit $p$-volume is preserved under dimensional oxidation, this provides a microscopic interpretation for the entropy of the Reissner-Nordstrøm black holes in $D=5$ [17] and $D=4$ [24, 25]. It is interesting to note that although the corresponding building blocks, namely $\Delta=4$ black holes, have vanishing entropy, these particular bound states, i.e. the Reissner-Nordstrøm black holes, nevertheless have nonvanishing entropy. On the other hand, all the other bound states, regular or singular, have vanishing entropy. However, it is worth remarking that all the regular bound states, if extrapolated to the near-extremal regime, satisfy the ideal-gas relation $S \sim T^{p}$ between their entropy and temperature, which is consistent with the D-brane picture [26, 27]. This relation is not satisfied, however, by the singular $p$-brane solutions. It was argued in [27] that if the singularities of the solutions were regulated by quantum effects, the classically-singular $p$-branes would also satisfy this ideal-gas relation, opening up the possibility of providing a microscopic interpretation of the entropy for all $p$-branes. 


\section{Multi-center solitons and intersecting $p$-branes}

Multi-center solutions provide a way of relating $p$-branes in different dimensions via the vertical dimension reduction procedure. The procedure involves two stages [16], namely an integration over a continuum of charges of the multi-center solutions distributed uniformly over lines, planes or hyperplanes, followed by an ordinary Kaluza-Klein reduction over the resulting Killing directions. It was shown in [12, 16] that at the intermediate stage, the metric configuration in the higher dimension can sometimes be interpreted as a special case of intersecting $p$-branes. For example, the membrane in $D=9$ can be viewed as a multi-center membrane in $D=11$, with the charges lying on a plane in the transverse space. Remarkably, it was shown that this plane can be elevated to the world-volume spatial dimensions of another elementary membrane in a more general solution [12]. This demonstrates that the membrane in 9 dimensions can be viewed as two intersecting membranes in $D=11$. This procedure provides an 11-dimensional interpretation of a large class of solutions, namely supersymmetric $p$-brane solutions whose charges are carried by the field strengths derived from the 4 -form in $D=11$. If a single M-brane is regarded as a special case of intersecting M-branes, these $p$-brane solitons in lower dimensions can be categorised in two classes:

1. The Kaluza-Klein dimensional reduction of intersecting M-branes. Examples are the $N$-charge black holes in $D=9,7,5$ and 3 with $N=1,2,3$ and 4 respectively. These black holes can be viewed as $N$ intersecting membranes in $D=11$, which preserve $2^{-N}$ of the supersymmetry 11, 12, 14, 16]. Another example is provided by the dyonic string in $D=6$, which can be viewed as a membrane intersecting a 5 -brane in $D=11$, which was constructed in [12].

2. Vertical dimensional reduction of intersecting M-branes. An example is the membrane in $D=10$, which can be viewed as the multi-center membrane in $D=11$ with its charges distributed uniformly on the extra dimension. Another example is the 2-charge black hole in $D=6$ that preserves $1 / 4$ of the supersymmetry. It can be viewed as two intersecting membranes in $D=11$, with the charges lying uniformly along a straight line.

In the lower dimensions, there also exist many other supersymmetric solitons whose charges are carried by the field strength derived from the vielbein, rather than from the 4form field strength in $D=11$. These solitons, together with the ones discussed above, form multiplets under the U duality symmetry. In this section, we shall discuss these solutions 
from the 11-dimensional point of view. We shall show that can be viewed in $D=11$ as boosted intersecting M-branes that carry momenta.

Let us first take black holes in $D=9$ as an example. There are in total three 2 -forms in $D=9: \mathcal{F}^{(1)}$ and $\mathcal{F}^{(2)}$ coming from the vielbein, and $F^{(12)}$ coming from the 4 -form in $D=11$. There are two two-scalar black holes, one using the 2-form field strength $F^{(12)}$ and $\mathcal{F}^{(1)}$, the other using $F^{(12)}$ and $\mathcal{F}^{(2)}$ 9. We shall call them A-type and B-type black holes respectively. Both solutions preserve $1 / 4$ of the supersymmetry. In fact they form a doublet under the Weyl group $S_{2}$ of the U duality group $S L(2, Z)$ in $D=9$ [28]. The metrics for the two solutions are identical, given by

$$
d s_{9}^{2}=-(H \widetilde{H})^{-\frac{6}{7}} d t^{2}+(H \widetilde{H})^{\frac{1}{7}} d y^{m} d y^{m}
$$

where $H$ is an harmonic function in the 8-dimensional transverse space associated with the field strength $F^{(12)}$, and $\widetilde{H}$ is an independent harmonic function associated with $\mathcal{F}^{(1)}$ or $\mathcal{F}^{(2)}$ in the A-type or B-type black holes respectively. We shall first study them from the 10-dimensional perspective. Since the 2-form field strength $\mathcal{F}^{(1)}$ exists already in $D=10$, the two-scalar A-type black hole, upon oxidation to 10 dimensions, gives rise to a string intersecting a black hole, with metric given by

$$
d s_{10}^{2}=-H^{-\frac{3}{4}} \widetilde{H}^{-\frac{7}{8}} d t^{2}+H^{-\frac{3}{4}} \widetilde{H}^{\frac{1}{8}} d z_{2}^{2}+H^{\frac{1}{4}} \widetilde{H}^{\frac{1}{8}} d y^{m} d y^{m}
$$

This interpretation can be seen by looking at the two limiting cases where either $H=1$, in which case (19) describes a multi-center black hole with its charges distributed uniformly along $z_{2}$, or $\widetilde{H}=1$, in which case it describes an isotropic string. Consideration of the generic interpolating solutions, where $H$ and $\widetilde{H}$ are both non-trivial, then completes the interpretation. Note that the string and the black hole share a common time coordinate and a common transverse subspace. The two-scalar B-type black hole, on the other hand, is quite different from the 10-dimensional point of view. Its metric is given by

$$
d s_{10}^{2}=-H^{-\frac{3}{4}} \widetilde{H}^{-1} d t^{2}+H^{-\frac{3}{4}} \widetilde{H}\left(d z_{2}+\mathcal{A}^{(2)}\right)^{2}+H^{\frac{1}{4}} d y^{m} d y^{m}
$$

This metric describes a boosted string carrying a momentum related to the charge carried by $\mathcal{F}^{(2)}=d \mathcal{A}^{(2)}$. The solution is analogous to the boosted dyonic string in $D=6$ [17, which is the dimensional oxidation of the 3-charge black hole in $D=5$.

We have seen that the two two-scalar black holes in $D=9$, which form a doublet under the $D=9$ Weyl U duality, have a very different interpretation in $D=10$. The A-type black hole gives rise to an intersection of a string and a black hole in $D=10$, whilst the B-type 
gives rise to a boosted string. Such a difference reflects the fact that $D=10$ is not the fundamental dimension. If we further oxidise the $D=10$ solutions to $D=11$, we obtain the two metrics

$$
\begin{aligned}
& \text { A-type : } \quad d s_{11}^{2}=-H^{-\frac{2}{3}} \widetilde{H}^{-1} d t^{2}+H^{-\frac{2}{3}} \widetilde{H}\left(d z_{1}+\mathcal{A}^{(1)}\right)^{2}+H^{-\frac{2}{3}} d z_{2}^{2}+H^{\frac{1}{3}} d y^{m} d y^{m}, \\
& \text { B-type }: \quad d s_{11}^{2}=-H^{-\frac{2}{3}} \widetilde{H}^{-1} d t^{2}+H^{-\frac{2}{3}} d z_{1}^{2}+H^{-\frac{2}{3}} \widetilde{H}\left(d z_{2}+\mathcal{A}^{(2)}\right)^{2}+H^{\frac{1}{3}} d y^{m} d y^{m} .
\end{aligned}
$$

Thus both the A-type and B-type black holes in $D=9$ can be viewed as boosted membranes in $D=11$, but carrying momentum in the two different spatial world-volume directions $z_{1}$ and $z_{2}$. The Weyl $\mathrm{U}$ duality in $D=9$ can be reinterpreted as a duality symmetry $z_{1} \leftrightarrow z_{2}$ in $D=11$. It is interesting that the manifest $\mathrm{U}$ Weyl duality, which is present in $D=9$, is somewhat obscured upon oxidation to $D=10$, but is restored in $D=11$. This can be taken as an indication that M-theory plays a more fundamental role than string theory.

The picture is more complicated in lower dimensions, where the $\mathrm{U}$ duality group is larger. For example, there are two kinds of 3-charge black holes in $D=5$, one of which involves field strengths coming only from the 4 -form in $D=11$, while the other involves a field strength coming from the metric. Upon oxidation the former becomes three intersecting membranes in $D=11$, with the metric 12 .

$$
\begin{aligned}
d s_{11}^{2}= & -\left(H_{1} H_{2} H_{3}\right)^{-\frac{2}{3}} d t^{2}+H_{1}^{-\frac{2}{3}}\left(H_{2} H_{3}\right)^{\frac{1}{3}}\left(d z_{1}^{2}+d z_{2}^{2}\right)+H_{2}^{-\frac{2}{3}}\left(H_{1} H_{3}\right)^{\frac{1}{3}}\left(d z_{3}^{2}+d z_{4}^{2}\right) \\
& +H_{3}^{-\frac{2}{3}}\left(H_{1} H_{2}\right)^{\frac{1}{3}}\left(d z_{5}^{2}+d z_{6}^{2}\right)+\left(H_{1} H_{2} H_{3}\right)^{\frac{1}{3}}\left(d y_{1}^{2}+\cdots+d y_{4}^{2}\right)
\end{aligned}
$$

where $H_{1}, H_{2}$ and $H_{3}$ are the harmonic functions associated with $F^{(12)}, F^{(34)}$ and $F^{(56)}$ respectively. On the other hand, we find that the latter metric becomes

$$
\begin{aligned}
d s_{11}^{2}= & -H_{1}^{-\frac{2}{3}} H_{2}^{-\frac{1}{3}} H_{3}^{-1} d t^{2}+H_{1}^{\frac{1}{3}} H_{2}^{-\frac{1}{3}}\left(d z_{1}^{2}+\cdots+d z_{4}^{2}\right)+H_{1}^{-\frac{2}{3}} H_{2}^{\frac{2}{3}} d z_{5}^{2} \\
& +H_{1}^{-\frac{2}{3}} H_{2}^{-\frac{1}{3}} H_{3}\left(d z_{6}+\mathcal{A}^{(6)}\right)^{2}+H_{1}^{\frac{1}{3}} H_{2}^{\frac{2}{3}}\left(d y_{1}^{2}+\cdots+d y_{4}^{2}\right)
\end{aligned}
$$

where in this example, $H_{1}, H_{2}$ and $H_{3}$ are the harmonic functions associated with $F^{(56)}$, $* F^{(5)}$ and $\mathcal{F}^{(6)}$ respectively. This describes a boosted membrane intersecting a 5-brane. (Note that Kaluza-Klein dimensional reduction of (23) to $D=6$ gives rise to the boosted dyonic string constructed in [17].) The two 3 -charge black holes in $D=5$ are related by $\mathrm{U}$ duality; in fact they belong to the same multiplet of the U Weyl group [28]. However, as we have seen, upon oxidation to $D=11$ the $\mathrm{U}$ duality is obscured, unlike the example of $D=9$ black holes that we discussed previously. It is tempting to speculate that manifest $\mathrm{U}$ duality might be restored in a yet higher dimension. 
Having illustrated the procedure with two examples, we can easily generalise these results to include all the supersymmetric $p$-branes in lower dimensions, in which some of the charges are carried by the field strengths coming from the vielbein. We shall categorise this type of $p$-brane in the third class:

3. Kaluza-Klein or vertical dimensional reduction of boosted intersecting M-branes.

\section{Conclusions and discussions}

In this paper, we constructed multi-scalar multi-center $p$-brane solutions, which are generalisations of supersymmetric single-scalar solutions with $\Delta=4 / N=2,4 / 3,1$, etc. The solutions carry $N$ independent sets of charges $k_{i}^{\alpha},(1 \leq \alpha \leq N)$, which can be located at different points $\vec{y}_{i}^{\alpha}$ in the transverse space. In the vicinity of each charge, the solution approaches a single-scalar solution with $\Delta=4$. If these locations coincide, the solution reduces to an isotropic multi-scalar solution. Furthermore, if the charges are set equal, it becomes a single-scalar solution with $\Delta=4 / N$. The multi-center solutions preserve the same fraction of the supersymmetry as their isotropic limits where the centers coincide. Thus we conclude that all supersymmetric isotropic single-scalar $p$-branes, and all their multi-scalar generalisations, can be viewed as bound states of supersymmetric $\Delta=4$ single-scalar building blocks. In addition, there are certain examples of $p$-branes with $\Delta=4 / N$ for $N \geq 4$ that do not have supersymmetry, owing to sign-reversals of some of the charges. These too can be interpreted as bound states of the same basic building blocks. All the building blocks are singular at the horizon, in that the scalar fields, field strengths and curvature diverge there. Interestingly, some of their bound states are nevertheless regular.

We also discussed the close relationship between vertical dimensional reduction and the description of intersecting M-branes. Consider a multi-center $p$-brane in $D$ dimensions whose charges lie uniformly on an $n$-dimensional hyperplane in the transverse space. This is the first stage in a vertical dimensional reduction to a $p$-brane in $(D-n)$ dimensions. On the other hand, in certain cases the hyperplane can be promoted to the status of being the spatial world-volume of a more general solution. Thus vertical dimension reduction provides a way of interpreting certain lower-dimensional $p$-branes as intersections of extended objects in higher dimensions. We showed that all supersymmetric $p$-branes in lower dimensions can be categorised by three classes. The first and second classes involve $p$-branes whose charges are all carried by those field strengths that are derived by dimensional reduction from the 4 -form in $D=11$. These solutions can be viewed as either Kaluza-Klein or vertical 
dimensional reductions of certain intersecting M-branes in $D=11$. The third class of $p$ branes have some charges that are carried by field strengths coming from the vielbein. These solutions can be regarded as Kaluza-Klein or vertical dimensional reductions of boosted intersecting M-branes.

Another interesting case that arises from the multi-scalar multi-center solutions is if each field strength carries charges distributed uniformly along different subspaces of the transverse space. In particular, these subspaces can be non-overlapping. For example, in the two-scalar black hole solution (18), the electric charge $q$ carried by $F^{(12)}$ can be chosen to lie uniformly in the $\left(y_{1}, y_{2}, y_{3}\right)$ hyperplane, while the electric charge $\tilde{q}$ carried by $\mathcal{F}^{(1)}$ or $\mathcal{F}^{(2)}$ can be chosen to lie uniformly in the $\left(y_{4}, \ldots, y_{8}\right)$ hyperplane. If $q$ is set to zero, the resulting metric configuration describes the Kaluza-Klein oxidation of a black hole in six dimensions. On the other hand, if the charge $\tilde{q}$ is zero, the solution is the oxidation of a black hole in four dimensions. These solutions are reminiscent of the previously-discussed intersecting $p$-branes, except that here the extended objects share a common world-volume but have non-coincident transverse spaces.

\section{Acknowledgement}

We are grateful to M.J. Duff, J. Rahmfeld and K.S. Stelle for useful discussions.

\section{References}

[1] M.J. Duff and K.S. Stelle, Multi-membrane solutions of $D=11$ supergravity, Phys. Lett. B253 (1991) 113.

[2] R. Güven, Black p-brane solitons of $D=11$ supergravity theory, Phys. Lett. B276 (1992) 49.

[3] H. Lü and C.N. Pope, p-brane solitons in maximal supergravities, hep-th/9512012, to appear in Nucl. Phys. B.

[4] M.J. Duff and J. Rahmfeld, Massive string states as extreme black holes, Phys. Lett. B345 (1995) 441.

[5] A. Sen, Black hole solutions in heterotic string theory on a torus, Nucl. Phys. B440 (1995) 421; Extremal black holes and elementary string states, Mod. Phys. Lett. A10 
(1995) 2081; A note on marginally stable bound states in Type II string theory, hepth/9510229.

[6] M.J. Duff, J.T. Liu and J. Rahmfeld, Four dimensional string/string/string triality, Nucl. Phys. B459 (1996) 125.

[7] M. Cvetic and A.A. Tseytlin, General class of BPS saturated dyonic black holes as exact superstring solutions, Phys. Lett. B366 (1996) 95.

[8] J. Rahmfeld, Extremal black holes as bound states, Phys. Lett. B372 (1996) 198.

[9] H. Lü and C.N. Pope, Multi-scalar p-brane solitons, hep-th/9512153, to appear in Int. J. Mod. Phys. A.

[10] R.R. Khuri and T. Ortin, A non-supersymmetric dyonic extremal Reissner-Nordstrøm black hole, hep-th/9512178.

[11] G. Papadopoulos and P.K. Townsend, Intersecting M-branes, hep-th/9603087.

[12] A.A. Tseytlin, Harmonic superpositions of M-theory, hep-th/9604035.

[13] I.R. Klebanov and A.A. Tseytlin, Intersecting M-branes as four-dimensional black holes, hep-th/9604166.

[14] J.P. Gauntlett, D.A. Kastor and J. Traschen, Overlapping Branes in M-theory, hepth/9604179.

[15] V. Balasubramanian and F. Larsen, On D-branes and black holes in four dimensions, hep-th/9604189.

[16] H. Lü, C.N. Pope and K.S. Stelle, Vertical versus diagonal dimensional reduction for p-branes, CTP TAMU-18/96, hep-th/9605082.

[17] A. Strominger and C. Vafa, Microscopic origin of Bekenstein-Hawking entropy, hepth/9601029.

[18] H. Lü, C.N. Pope and K.W. Xu, Liouville and Toda solitons in M-theory, hepth/9604058.

[19] M.J. Duff, S. Ferrara, R.R. Khuri and J. Rahmfeld, Supersymmetry and dual string solitons, Phys. Lett. B356 (1995) 479. 
[20] M.J. Duff and J.X. Lu, Black and super p-branes in diverse dimensions, Nucl. Phys. B416 (1994) 301.

[21] M.J. Duff, H. Lü and C.N. Pope, Heterotic phase transitions and singularities of the gauge dyonic string, hep-th/9603037, to appear in Phys. Lett. B.

[22] G.T. Horowitz and A. Strominger, Black strings and p-branes, Nucl. Phys. B360 (1991) 197.

[23] M.J. Duff and J.X. Lu, The self-dual type IIB superthreebrane, Phys. Lett. B273 (1991) 409.

[24] C.V. Johnson, R.R. Khuri and R.C. Myers, Entropy of 4-D extremal black holes, hepth/9603061.

[25] A. Strominger and J. Maldacena, Statistical entropy of four-dimensional extremal back holes, hep-th/9603060.

[26] I.R. Klebanov and A.A. Tseytlin, Entropy of near-extremal black p-branes, hepth/9604089.

[27] H. Lü, S. Mukherji, C.N. Pope and J. Rahmfeld, Loop-corrected entropy of nearextremal dilatonic p-branes, hep-th/9604127.

[28] H. Lü, C.N. Pope and K.S. Stelle, Weyl group invariance and p-brane multiplets, hepth/9602140, to appear in Nucl. Phys. B. 